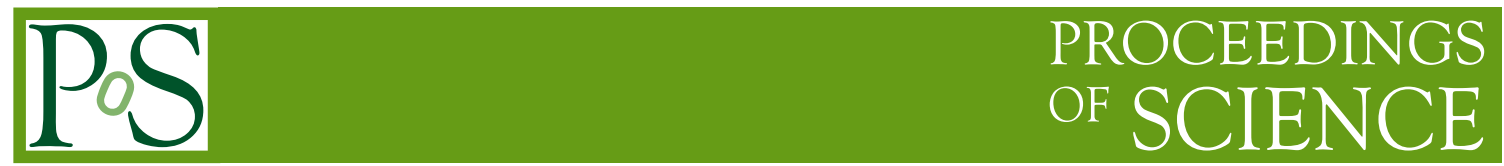

\title{
New constraints on heavy neutral leptons coming from oscillation data analysis and precision $e^{+} e^{-}$physics
}

\author{
Wojciech Flieger ${ }^{a, *}$ and Krzysztof Grzanka ${ }^{a}$ \\ ${ }^{a}$ Institute of Physics, University of Silesia, \\ Katowice, Poland \\ E-mail: woj.flieger@gmail.com, krzysztof.grzanka@us.edu.pl
}

\begin{abstract}
The current experimental data does not exclude the possibility that additional sterile neutrinos exist. We discuss two methods to determine active-sterile neutrino mixing. Firstly, singular values provide a comprehensive description of the mixing phenomena. By using them, we get the stringent bounds for the active-sterile mixing in the scenario with one additional neutrino. Secondly, we describe a simplified model with a sterile neutrino to show a sensitivity of the invisible Z-boson decay to the sterile neutrino mixings. In the end, we outline the precise analysis of the light-heavy mixings coming from the Z-boson decay taking into account the first-order radiative corrections.
\end{abstract}

40th International Conference on High Energy physics - ICHEP2020

July 28 - August 6, 2020

Prague, Czech Republic (virtual meeting)

\footnotetext{
${ }^{*}$ Speaker
} 


\section{Introduction}

Neutrino physics provides many challenging questions. Amongst them is that concerning the number of neutrino types in nature. The current status is that there are three types (flavours) $v_{\alpha}^{(f)}$ where $\alpha=e, \mu, \tau$ of neutrinos which are composed of three massive states $v_{i}^{(m)}$ where $i=1,2,3$. This composition of flavours states as a combination of massive states is known as neutrino mixing and can be viewed as a transition between two orthogonal state bases given by the unitary matrix called the PMNS mixing matrix, i.e., $v_{\alpha}^{(f)}=\sum_{i=1}^{3}\left(U_{\mathrm{PMNS}}\right)_{\alpha i} v_{i}^{(m)}$. However there are experimental and theoretical clues indicating that at least one additional neutrino is necessary. If it is the case the new neutrino fields $\tilde{v}_{\beta(j)}$ where $\beta(j)=1, \ldots, n_{r}$ can mix with the standard neutrinos and signals of such mixing must be visible as a deviation from unitarity of the PMNS mixing matrix wherein the complete mixing matrix preserves unitarity

$$
\left(\begin{array}{c}
v_{\alpha}^{(f)} \\
\tilde{v}_{\beta}^{(f)}
\end{array}\right)=\left(\begin{array}{cc}
U_{l l} & U_{l h} \\
U_{h l} & U_{h h}
\end{array}\right)\left(\begin{array}{c}
v_{i}^{(m)} \\
\tilde{v}_{j}^{(m)}
\end{array}\right) .
$$

In this paper we will discuss methods to estimate the active-sterile mixing $U_{l h}$. The emphasis will be put on the $3+1$ scenarios, i.e., the scenario with one additional neutrino.

\section{Estimation of the active-sterile mixing}

\subsection{Singular values based method}

In [1] an interesting connection between mixing matrices and quantities known as singular values has been pointed out. It turn out that all physical mixing matrices must be contractions, i.e., matrices with the largest singular value less than or equal to one 1 , where singular values of a given matrix $U \in \mathbb{C}^{n \times n}$ are defined as the positive square roots of the eigenvalues of $U U^{\dagger}$, i.e., $s_{i}(A)=\sqrt{\lambda_{i}\left(U U^{\dagger}\right)}$ for $\mathrm{i}=1,2, \ldots, \mathrm{n}$. This characterization allows us to treat both SM and BSM scenarios in a uniform way. Moreover all physically interesting situations can be described by 3-dimensional PMNS mixing matrices. It is so since all physically admissible 3-dimensional mixing matrices belong to the convex hull spanned by the 3-dimensional unitary matrices

$$
\begin{gathered}
\Omega:=\operatorname{conv}\left(U_{\mathrm{PMNS}}\right)=\left\{\sum_{i=1}^{m} \alpha_{i} U_{i} \mid U_{i} \in U(3), \alpha_{1}, \ldots, \alpha_{m} \geq 0, \sum_{i=1}^{m} \alpha_{i}=1,\right. \\
\left.\theta_{12}, \theta_{13}, \theta_{23} \text { and } \delta \text { given by experimental values }\right\}
\end{gathered}
$$

The connection between the $\Omega$ region and BSM scenarios follows from the fact that the only contraction can be extended to a larger unitary matrix and this procedure is called a unitary dilation. However, the minimal dimension of such extension is not arbitrary but depends on the number of singular values strictly less than one. This allows us to divide the $\Omega$ region into four disjoint subsets according to the minimal number of additional neutrinos

$$
\begin{array}{ll}
\Omega_{1}: & 3+1 \text { scenario: } \Sigma=\left\{\sigma_{1}=1.0, \sigma_{2}=1.0, \sigma_{3}<1.0\right\}, \\
\Omega_{2}: & 3+2 \text { scenario: } \Sigma=\left\{\sigma_{1}=1.0, \sigma_{2}<1.0, \sigma_{3}<1.0\right\}, \\
\Omega_{3}: & \text { 3+ 3 scenario: } \Sigma=\left\{\sigma_{1}<1.0, \sigma_{2}<1.0, \sigma_{3}<1.0\right\}, \\
\Omega_{4}: & \text { PMNS scenario: } \Sigma=\left\{\sigma_{1}=1, \sigma_{2}=1, \sigma_{3}=1\right\} .
\end{array}
$$


Thus, we can study each scenario separately. In order to estimate the active-sterile mixing $U_{l h}$ we must study extensions of these subsets. This can be done with help of a cosine-sine (CS) decomposition of block unitary matrices [2]

$$
U \equiv\left(\begin{array}{cc}
U_{l l} & U_{l h} \\
U_{h l} & U_{h h}
\end{array}\right)=\left(\begin{array}{cc}
W_{1} & 0 \\
0 & W_{2}
\end{array}\right)\left(\begin{array}{cc|c}
I_{m-n} & 0 & 0 \\
0 & C & -S \\
\hline 0 & S & C
\end{array}\right)\left(\begin{array}{cc}
Q_{1}^{\dagger} & 0 \\
0 & Q_{2}^{\dagger}
\end{array}\right),
$$

where $W_{1}, W_{2}$ and $Q_{1}, Q_{2}$ are unitary matrices and $C$ and $S$ are diagonal matrices which satisfy $C^{2}+S^{2}=I$. In case of one additional neutrino the CS decomposition takes the following form

$$
\left(\begin{array}{cc}
U_{l l} & U_{l h} \\
U_{h l} & U_{h h}
\end{array}\right)=\left(\begin{array}{cc}
W_{1} & 0 \\
0 & W_{2}
\end{array}\right)\left(\begin{array}{ccc|c}
1 & 0 & 0 & 0 \\
0 & 1 & 0 & 0 \\
0 & 0 & c & -s \\
\hline 0 & 0 & s & c
\end{array}\right)\left(\begin{array}{cc}
Q_{1}^{\dagger} & 0 \\
0 & Q_{2}^{\dagger}
\end{array}\right) .
$$

The $U_{l h}$ is given by

$$
U_{l h}=W_{1} S_{12} Q_{2}^{\dagger},
$$

where $W_{1} \in \mathbb{C}^{3 \times 3}$ is unitary, $S_{12}=(0,0,-s)^{T}$ and $Q_{2}=e^{i \theta}, \theta \in(0,2 \pi]$. Then by taking exact values of $W_{1}$ we obtain the analytic formula for the active-sterile mixing

$$
\left|U_{i 4}\right|=\left|w_{i 3}\right| \cdot\left|\sqrt{1-\sigma_{3}^{2}}\right|, \quad i=e, \mu, \tau .
$$

For example in case of heavy sterile neutrino $(m>$ EW) we get [3]

$$
\begin{aligned}
& \left|U_{e 4}\right| \in[0,0.021], \quad\left|U_{\mu 4}\right| \in[0.00013,0.021], \quad\left|U_{\tau 4}\right| \in[0.0115,0.075] . \\
& \left|U_{e 4}\right| \leq 0.041 \text { [4], }\left|U_{\mu 4}\right| \leq 0.030 \text { [4], } \quad\left|U_{\tau 4}\right| \leq 0.087 \text { [4]. }
\end{aligned}
$$

Results for other massive scenarios are presented in [3].

\subsection{Z-boson decay}

The Z-boson decay width is an important observable in neutrino research providing constrains on masses and mixings. Its invisible part is connected to the number of active neutrinos by

$$
N_{v}=\left(\frac{\Gamma_{\text {inv }}}{\Gamma_{\text {lept }}}\right)^{\text {meas }} /\left(\frac{\Gamma_{\nu \bar{v}}}{\Gamma_{\text {lept }}}\right)^{S M}
$$

This number was intensively studied in the LEP experiment and the number of neutrino families was established to be $[5,6]$

$$
N_{v}=2.9840 \pm 0.0082
$$

Recent update on precision calculations gave better estimate of this number [7, 8]

$$
N_{v}=2.9963 \pm 0.0074 .
$$

The Jarlskog theorem [9] states that if additional neutrinos exist then $N_{v}$ is less than three. The present status agrees with that and we will use $N_{v}$ to estimate bounds for the active-sterile mixing. 


\subsubsection{A toy model}

The simplest extension of the Standard Model by one neutrino is given by the model where the mass matrix takes the seesaw-like form

$$
\mathcal{L}_{v}^{M}=-\frac{1}{2} \bar{N}\left(\begin{array}{cccc}
0 & 0 & 0 & a_{1} \\
0 & 0 & 0 & a_{2} \\
0 & 0 & 0 & a_{3} \\
a_{1} & a_{2} & a_{3} & M
\end{array}\right) N^{C}+H . c .
$$

where $N=\left(v_{1}, v_{2}, v_{3}, v_{4}\right)$. Such a model was studied in [9, 10]. However, from the point of view of the present experimental knowledge, it serves just as a toy model since it leads to two massless neutrinos. Nevertheless, due to its simplicity it is a good starting point to study the sensitivity of the updated result for $N_{v}$ (14) to the active-sterile mixing. For this model, the nonstandard part of the invisible Z-decay can be isolated giving

$$
N_{v}-2=\frac{1}{(x+y)^{2}}\left[x^{2} F(y)+y^{2} F(x)+2 x y G(x, y)\right] \text {, }
$$

where $x=\frac{m_{4}}{M_{Z}}$ and $y=\frac{m_{3}}{M_{Z}}$ with $m_{3,4} \leq M_{Z} / 2$. The active-sterile mixing in this case is given just by $\sin \alpha$ which can be read from the following relation $y=x \tan ^{2} \alpha$ by calculating the slope of the line, which lies below experimentally determined limits Fig. 1.

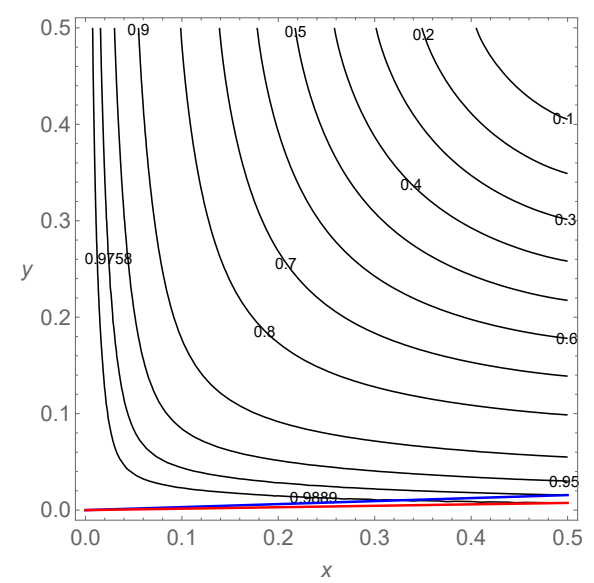

Figure 1: The slope of the blue and red lines represents $\tan ^{2} \alpha$ for the previous and updated result for $N_{\nu}$, equations (13) and (14), respectively.

This procedure leads to the following results

$$
\begin{aligned}
& \mathrm{LEP}: N_{v}=2.9840 \pm 0.0082 \rightarrow \sum_{i}\left|U_{i 4}\right| \equiv \sin \alpha<0.174 \quad i=e, \mu, \tau . \\
& \mathrm{NEW}: N_{v}=2.9963 \pm 0.0074 \rightarrow \sum_{i}\left|U_{i 4}\right| \equiv \sin \alpha<0.121 \quad i=e, \mu, \tau .
\end{aligned}
$$

The updated result provides the significant improvement in the estimation of the active-sterile mixing which motivates further study of realistic scenarios. 


\subsubsection{A realistic model}

The presence of sterile neutrinos impacts the invisible Z-decay width as they mix with the active neutrinos. We discuss the influence of the sterile neutrinos on $\Gamma_{i n v}$ in models with additional $n_{r}$ right-handed neutrinos. The existence of sterile neutrinos manifests itself both at tree and loop level. In $[11,12]$ the impact of sterile neutrinos to the electro-weak pseudo observable (EWPO) has been discussed in the framework of the seesaw scenario. Due to their presence the PMNS mixing matrix is no longer unitary and this deviation from unitarity is the main contribution to EWPO coming from sterile neutrinos at the tree level. Such deviation can be parametrized as $\epsilon_{\alpha} \equiv \sum_{i \geq 4}\left|U_{\alpha i}\right|^{2}$ where $\alpha=e, \mu, \tau$. The contribution of right-handed neutrinos at the loop level modify the oblique corrections $S, T, U$ which can be expressed in term of masses and mixings of the sterile neutrinos. Taking this into account the following formula is valid [11, 12]

$$
\left(\frac{\Gamma_{\text {inv }}}{\Gamma_{\text {lept }}}\right)^{\text {meas }} /\left(\frac{\Gamma_{\text {inv }}}{\Gamma_{\text {lept }}}\right)^{S M}=1-0.76\left(\epsilon_{e}+\epsilon_{\mu}\right)-0.67 \epsilon_{\tau}-0.0015 T \text {. }
$$

The influence of the sterile neutrinos to the EWPO at the tree level can also be studied by invoking different parametrization of the deviation from unitarity of the PMNS mixing matrix [13,14]. Such a non-unitary mixing matrix $U_{l l}$ can be written as the product of unitary matrix $U$ and positive-definite Hermitian matrix $\eta$, i.e., $U_{l l}=(I-\eta) U$ providing

$$
\Gamma_{i n v}=\frac{G_{F} M_{Z}^{3} \sum_{i j}\left|U_{l l}^{\dagger} U_{l l}\right|^{2}}{12 \sqrt{2} \pi} \simeq \frac{G_{F} M_{Z}^{3}}{12 \sqrt{2} \pi}\left(3-\left(4 \eta_{\tau \tau}+\eta_{e e}+\eta_{\mu \mu}\right)\right) .
$$

Finally, the detailed discussion [15] of importance of loop corrections in the estimation of activesterile mixing in the seesaw scenario revealed that they can be relevant in some parts of the parameter space. The expression for $\Gamma_{i n v}$ with the loop correction takes the following form

$$
\Gamma_{i n v}=\sum_{i, j=1}^{3} \frac{G_{F} M_{Z}^{3} \rho}{24 \sqrt{2} \pi}\left(\mathcal{Z}_{i j}+\mathcal{Z}_{j i}\right)
$$

where $\rho$ contains the SM loop corrections to the process and

$$
\mathcal{Z}_{i j}=\left|C_{i j}\right|^{2}\left(1+\delta_{Z}^{u n i v}\right)+2 \operatorname{Re}\left[C_{i j}^{*}\left(\delta_{i j}^{C T Z}+\mathcal{V}_{i j}^{Z}\right)\right],
$$

with $C_{i j}=\sum_{\alpha=e, \mu, \tau} U_{\alpha i}^{*} U_{\alpha j}$ and $\delta_{i j}^{C T Z}$ and $\mathcal{V}_{i j}^{Z}$ the lepton-flavour-dependent counterterm and vertex interference, respectively.

As the dilation procedure allows us to discuss $3+1$ and $3+2$ scenarios independently, the goal for the future work is to estimate new limits to the active-sterile mixings taking into account 1-loop corrections to the Z-boson decay due to sterile neutrinos and the updated result for the number of active neutrinos $N_{\nu}$.

Acknowledgments. We would like to thank Janusz Gluza and Kamil Porwit for useful comments and discussions. The work is supported by the Polish National Science Centre under grant no. 2017/25/B/ST2/01987 and COST Action CA16201 PARTICLEFACE. 


\section{References}

[1] K. Bielas, W. Flieger, J. Gluza, M. Gluza, Neutrino mixing, interval matrices and singular values, Phys. Rev. D 98 (5) (2018) 053001. doi : 10 .1103/PhysRevD . 98.053001.

[2] A. Allen, D. Arceo, Corporate Author: Space and Naval Warfare Systems Center San Diego CA, Matrix Dilations via Cosine-Sine Decomposition, Defense Technical Information Center. https://apps.dtic.mil/dtic/tr/fulltext/u2/a446226.pdf (2006).

[3] W. Flieger, J. Gluza, K. Porwit, New limits on neutrino non-unitary mixings based on prescribed singular values, JHEP 03 (2020) 169. doi : 10 . 1007/JHEP03(2020) 169.

[4] J. de Blas, Electroweak limits on physics beyond the Standard Model, EPJ Web Conf. 60 (2013) 19008. arXiv: 1307.6173, doi:10.1051/epjconf/20136019008.

[5] S. Schael, et al., Precision electroweak measurements on the $Z$ resonance, Phys. Rept. 427 (2006) 257-454. arXiv:hep-ex/0509008, doi:10.1016/j . physrep.2005.12.006.

[6] V. A. Novikov, L. B. Okun, A. N. Rozanov, M. I. Vysotsky, Theory of $Z$ boson decays, Rept. Prog. Phys. 62 (1999) 1275-1332. doi : 10.1088/0034-4885/62/9/201.

[7] G. Voutsinas, E. Perez, M. Dam, P. Janot, Beam-beam effects on the luminosity measurement at LEP and the number of light neutrino species, Phys. Lett. B800 (2020) 135068. arXiv: 1908.01704, doi:10.1016/j . physletb.2019.135068.

[8] P. Janot, S. Jadach, Improved Bhabha cross section at LEP and the number of light neutrino species, Phys. Lett. B803 (2020) 135319. doi : 10 . 1016/j . physletb. 2020 . 135319.

[9] C. Jarlskog, Neutrino Counting at the $Z$ Peak and Right-handed Neutrinos, Phys. Lett. B241 (1990) 579-583. doi : 10.1016/0370-2693(90)91873-A.

[10] C. Escobar, O. Peres, V. Pleitez, R. Zukanovich Funchal, Constraints on singlet right handed neutrinos coming from the Z0 width, Phys. Rev. D 47 (1993) 1747-1750. arXiv: hep-ph/ 9212282, doi: 10.1103/PhysRevD.47.R1747.

[11] E. Akhmedov, A. Kartavtsev, M. Lindner, L. Michaels, J. Smirnov, Improving Electro-Weak Fits with TeV-scale Sterile Neutrinos, JHEP 05 (2013) 081. doi : $10.1007 /$ JHEP05 (2013) 081.

[12] L. Basso, O. Fischer, J. J. van der Bij, Precision tests of unitarity in leptonic mixing, EPL 105 (1) (2014) 11001. arXiv: 1310.2057, doi : 10.1209/0295-5075/105/11001.

[13] S. Antusch, O. Fischer, Non-unitarity of the leptonic mixing matrix: Present bounds and future sensitivities, JHEP 10 (2014) 094. arXiv: 1407.6607, doi : 10 . 1007/JHEP10 (2014) 094.

[14] E. Fernandez-Martinez, J. Hernandez-Garcia, J. Lopez-Pavon, Global constraints on heavy neutrino mixing, JHEP 08 (2016) 033. doi : 10 . 1007/JHEP08 (2016) 033.

[15] E. Fernandez-Martinez, J. Hernandez-Garcia, J. Lopez-Pavon, M. Lucente, Loop level constraints on Seesaw neutrino mixing, JHEP 10 (2015) 130. doi : 10 . 1007/JHEP10 (2015) 130. 